\title{
Ejercicio físico, parámetros nutricionales y composición corporal en pacientes en hemodiálisis
}

\author{
Anna Junqué Jiménez, Ester Tomás Bernabeu, Gorka Iza Pinedo, Inés Luceño Solé, Oscar Paz López, \\ Marisa Lavado Santpere
}

\section{Servei de Nefrología. Hospital de Terrassa. Consorci Sanitari Terrassa. Barcelona}

\section{Introducción:}

Los pacientes en hemodiálisis (HD) se caracterizan por una elevada pérdida muscular, disminución de la condición física y deteriorada calidad de vida. Escasos estudios acerca del ejercicio físico en HD en relación con la composición corporal han sido publicados hasta la fecha.

\section{Objetivos:}

Analizar si la introducción de un programa de ejercicio físico intradiálisis es capaz de mejorar la fuerza muscular, los parámetros nutricionales y la composición corporal en nuestros pacientes en HD.

\section{Material y métodos:}

Estudio prospectivo unicéntrico de 12 semanas de duración. Los pacientes incluídos realizaban un programa completo de ejercicio físico usando pelotas, pesas, bandas elásticas y cicloergómetros en las primeras dos horas de HD.Analizamos:

1. Parámetros bioquímicos nutricionales (Albúmina, prealbúmina, triglicéridos, colesterol total y fracciones, ferritina y Proteina C Reactiva).

2. Fuerza extensión máxima quadriceps (FEMQ) y handgrip (HG).

3. Tests Capacidad funcional:"Sit to stand to sit" (STS10) y "six-minutes walking test" (6MWT).

4. Composición corporal mediante biompedancia electrica (BIA).

\section{Resultados:}

Nueve pacientes en HD incluídos: $56 \%$ hombres. Edad media. 65.6 años y 48.6 meses en HD. 0bservamos una mejoría significativa $\left({ }^{*} p<0,05\right)$ en $F E M Q^{*}(17.2 \pm 11.9$ vs $21.1 \pm 12.5 \mathrm{~kg})$, $H G *(25.6 \pm 13.1$ vs $27.3 \pm 12.6 \mathrm{~kg}), \mathrm{STS} 10 *(23 \pm 6.7$ vs $20.3 \pm 5.9 \mathrm{sec})$ y $6 \mathrm{MWT}^{*}(418.6$ vs $439 \mathrm{~m})$. En relación a la composición corporal, observamos una tendencia a la disminución del \% de grasa corporal (29.4\%vs28.3\%) y a un incremento significativo del tejido musculoesquelético $(30.7 \%$ vs $31.9 \%, \mathrm{p}<0.05)$. Asímismo, observamos una relación inversa negativa del \% grasa corporal con el HG $(r=-0.79)$ y FEMQ $(r=-0.92)$. No se observaron cambios en los datos biqouímicos al finalizar el estudio.

\section{Conclusiones:}

1. A pesar de que la realización de ejercicio físico intradiálisis mejoró la fuerza muscular y la capacidad funcional, no observamos cambios en los datos bioquímicos nutricionales.

2. El ejercicio físico intradiálisis mejoró la composición corporal en los pacientes en HD de nuestro estudio.

3. No obstante, se requieren estudios prospectivos bien diseñados para determinar el potencial efecto beneficioso del ejercicio físico sobre la composición corporal en estos pacientes. 


\section{Referencias Bibliográficas}

1. Blake C, Codd MB, Cassidy A, O'Meara YM. Physical function, employment and quality of life in endstage renal disease. J Nephrol 2000;13(2):142-9.

2. Segura-Ortí E, Rodilla-Alama V, Lisón JF. Physiotherapy during hemodialysis: Results of a progressive resistance-training programa. Nefrología 2008;28(1):67-72.

3. Fitts SS, Guthrie MR, Blagg CR. Exercise coaching and rehabilitation counseling improve quality of life for predialysis and dialysis patients. Nephron 1999;82(2):115-21.

4. Cheema B, Abas H, Smith B, O'Sullivan A, Chan M, Patwardhan $A$,et al. Progressive exercise for anabolism in kidney disease (PEAK): A randomized, controlled trial of resistance training during hemodialysis. J Am Soc Nephrol 2007;18(5):1594-601.

5. Deligiannis A, Kouidi E, Tassoulas E, Gigis P, Tourkantonis A, Coats A. Cardiac effects of exercise rehabilitation in hemodialysis patients. Int $\mathrm{J}$ Cardiol 1999;70(3):253-66.
6. Goldberg AP, Geltman EM, Hagberg JM, Gavin JR 3rd, Delmez JA,Carney RM, et al. Therapeutic benefits of exercise training for hemodialysis patients. Kidney Int Suppl 1983;16:S303-9.

7. Harter HR, Goldberg AP. Endurance exercise training. An effective therapeutic modality for hemodialysis patients. Med Clin North Am 1985;69(1):159-75.

8. Parsons TL, Toffelmire EB, King-VanVlack CE. The effect of an exercise program during hemodialysis on dialysis efficacy, blood pressure and quality of life in end-stage renal disease (ESRD) patients. Clin Nephrol 2004;61(4):261-74.

9. Cupisti A, Capitanini A, Betti G, D'Alessandro C, Barsotti G. Assessment ofhabitual physical activity and energy expenditure in dialysis patients andrelationships to nutritional parameters. Clin Nephrol. 2011 Mar;75(3):218-25.

10. Johansen $K L$, Painter $P L$, Sakkas GK, Gordon $P$, Doyle J, Shubert T. Effects ofresistance exercise training and nandrolone decanoate on body composition and muscle function among patients who receive hemodialysis: A randomized, controlled trial. J Am Soc Nephrol. 2006 Aug;17(8):230714. Epub 2006 Jul 6. 